\title{
Screenings toxicologiques par LC-MS simple-Quad versus HPLC-DAD : bilan après un an de pratique en urgence hospitalière
}

\section{Toxicological screenings with $L C-M S$ versus $H P L C-D A D$ : one year of practice in emergency care}

\section{Olivier MATHIEU ${ }^{(1)}$, Sandrine GUTKNECHT ${ }^{(2)}$, Franck BARNAY(1), Jean-Marie BERTHEZENE(2), Anne-France BONGRAND(3), Jean-Claude MATHIEU-DAUDE ${ }^{(1) *}$}

(1) Service de Pharmacologie Médicale et Toxicologie - Hôpital Lapeyronie

(2) Service des Urgences - Hôpital Lapeyronie (3) Centre de référence de la mort subite du nourrisson - Hôpital Arnaud de Villeneuve

*Auteur à qui adresser la correspondance : Jean-Claude MATHIEU-DAUDE, CHU de Montpellier, 191, avenue du Doyen Gaston Giraud - 34295 MONTPELLIER Cedex 5 - Tél : 0467338321 - Fax : 0467336751

(Reçu le 17 janvier 2005 ; accepté après modifications le 18 avril 2005)

\section{RÉSUMÉ}

Nous rapportons le bilan d'une année d'application de la chromatographie liquide couplée à un détecteur de masse simple-quad (LC-MS) à des screenings toxicologiques dans le cadre de l'urgence hospitalière. Plus précisément ce type de screening a été appliqué à des cas de mort subite du nourrisson, des comas toxiques non renseignés par les bilans standards, et à une étude sur les bilans toxicologiques systématiques à l'accueil des Urgences. Les données analytiques obtenues ont été confrontées à celles obtenues par un screening conventionnel par chromatographie liquide couplée à un détecteur à barrette de diodes (HPLC-DAD).

L'analyse des données indique que le screening toxicolo-

\section{SUMMARY}

This work is a review of one year of practice in LC-MS single-quad toxicological screening for patients with toxicological etiology admitted in an emergency unit. Systematic research using this technique was applied in three situations : sudden infant death syndrom, comatose patients with toxicological etiology, patients included in a systematic study with the emergency admission unit. Analytical results were confronted with conventional LC-DAD screening. Spectral data from toxicological screening with LC-MS identify several xenobiotics and their metabolites in a single analytic step, compatible with time delay involved in emergency treatments. In addition, screening detection limits are generally 
gique par LC-MS permet l'identification formelle de nombreuses molécules et de leurs métabolites en une seule étape analytique simple dans un délai compatible avec l'urgence clinique. De plus la sensibilité de la méthode permet de détecter des doses infra-thérapeutiques. Cependant sa limite majeure réside dans la construction, à la charge de l'utilisateur; d'une bibliothèque spectrale spécifique suffisamment exhaustive pour les toxiques généralement rencontrés. Dans le cas de niolécules non répertoriées, le croisenent des données obtenues par l'utilisation conjointe des deux méthodes de screening, DAD et MS, conduit parfois à une identification.

Au terne d'un an d'expérience, nous avons placé la $L C-M S$ comme méthode analytique de première intention pour la réalisation des screenings toxicologiques urgents. L'optimisation du screening toxicologique par LC-MS doit permettre d'étendre le champ d'application de la méthode à d'autres besoins hospitaliers.

\section{MOTS-CLÉS}

Screening, dépistage, clinique, LC-MS, HPLC-DAD.

\section{Introduction}

Les screenings toxicologiques larges sont longtemps restés inaccessibles aux situations d'urgence du fait de l'absence de techniques de détections polyvalentes suffisamment sensibles. La chromatographie liquide couplée au détecteur à barrettes de diode (HPLC-DAD) et la constitution de bibliothèques dédiées ont permis un premier essor de ce type de screening. Cette technique permet de coupler une donnée chromatographique, un temps de rétention, avec un paramètre assez spécifique d'une molécule, son spectre UV. Cependant des composés pharmacochimiquement proches peuvent partager ces deux paramètres, rendant peu sûre l'identification et obligatoire une analyse complémentaire, d'où un délai important dans le rendu des résultats. De plus, la limite majeure réside dans le coefficient d'extinction spécifique des toxiques souvent trop faible pour permettre leur dépistage. Les méthodes automatisées de type immunoenzymatiques permettent de répondre très rapidement quant à la présence ou l'absence de tel ou tel type précis de toxique. Mais le panel des molécules accessibles par ces méthodes est restreint. La chromatographie phase gazeuse couplée à un détecteur de masse (GC-MS) permet l'identification formelle des toxiques, même pour des concentrations faibles. Cependant cette technique spécifique et sensible est limitée aux molécules thermostables et nécessite un lourd travail pré-analytique. La chromatographie liquide couplée à un détecteur de masse (LC-MS), réservée souvent à la quantification de molécules présentes à de très faibles concentrations, semble être une bonne candidate pour les screenings toxicologiques larges de par son champ d'application analytique (1). De plus la LC- as low as therapeutic ranges.

The main difficult' is to build a specific mass spectrum library for the most common toxic agents. In the case of a nomatch chromatographic peak, no chemical information is available from mass spectrum data; but shared data from $L C-M S$ and $L C-D A D$ will improve the identification.

In our laboratory, LC-MS is actually used in first intention for urgent toxicological screenings:

\section{KEY-WORDS}

Screening, detection, clinical care, LC-MS, HPLC-DAD.

MS ne nécessite qu'une étape pré-analytique simplifiée.

Nous présentons ici le bilan d'une année de screenings toxicologiques réalisés simultanément avec une HPLCDAD classique et une LC-MS simple quadripôle avec traitement informatique par déconvolution croisé avec une bibliothèque développée par le laboratoire. Un bilan immunoenzymatique standard a été réalisé dans tous les cas.

\section{Matériel et méthode}

Les screenings HPLC-DAD sont effectués sur une chaîne WATERS 600S avec une colonne C8 Symmetry $5 \mu \mathrm{m}, 4.6 \mathrm{X} 250 \mathrm{~mm}$ équipée d'un détecteur à barrette de diodes WATERS 996 avec un balayage $220-400 \mathrm{~nm}$. Le gradient de 32 minutes acétonitrile/tampon phosphate est celui préconisé par WATERS pour satisfaire aux conditions de la bibliothèque. Les screenings LCMS sont effectués sur une chaîne Hewlett-Packard 1100 Series avec un détecteur de masse G1946A. Un détecteur UV monochromatique VARIAN 9050 réglé à $254 \mathrm{~nm}$ est de plus placé en amont du détecteur de masse. La colonne utilisée est une colonne Zorbax Eclipse XDBC C8 5um, 4.6X150 mm. Le gradient est le même que celui de l'HPLC-DAD avec un tampon formiate. Le détecteur de masse est utilisé en mode électrospray positif avec une tension de cône à $120 \mathrm{~V}$ et une tension de capillaire à 4000V. L'acquisition est réalisée en mode scan avec un balayage de 50 à $600 \mathrm{~m} / \mathrm{z}$. Ces paramètres ont été déterminés lors d'une étude visant à obtenir sur notre plate-forme les spectres les plus pertinents pour constituer une bibliothèque spec- 
trale. Cette étude était dérivée de travaux déjà publiés $(4,5,6)$.

L'acétonitrile (SIGMA-ALDRICH) des phases mobiles et les réactifs utilisés pour l'extraction des plasmas : carbonate de sodium, chloroforme, isopropanol (PROLABO) sont de qualité HPLC. Le tampon phosphate $50 \mathrm{mM}$ est préparé en dissolvant $7,8 \mathrm{~g}$ de dihydrogénophosphate de sodium hydraté (MERCK) dans 1 litre d'eau milliQ. Le pH est ajusté à 3,8 avec de l'acide orthophosphorique (MERCK). Le tampon formiate $2 \mathrm{mM}$ est préparé en dissolvant $126 \mathrm{mg}$ de formiate d'ammonium (SIGMA-ALDRICH) dans 1 litre d'eau milliQ. Le pH est ajusté à 3,0 avec de l'acide formique (MERCK).

Les échantillons sanguins proviennent soit du Centre de référence de la mort subite du nourrisson, soit du département d'Anesthésie-Réanimation, soit du service des Urgences du CHU de Montpellier. Le sang prélevé sur héparinate de lithium, le plasma est récupéré après centrifugation à $3000 \mathrm{tr} / \mathrm{min}$ pendant 10 minutes.

Un premier bilan standard sur $100 \mu \mathrm{l}$ de plasma est effectué sur un automate Xpand de la série Dimension de DADE-BEHRING. Ce bilan comprend la recherche des benzodiazépines, de l'alcool, du phénobarbital, du paracétamol, des salicylés avec les kits DADE-BEHRING commercialisés avec l'automate. Les antidépresseurs imipraminiques sont recherchés avec un kit SYVA adapté à l'automate. Il s'agit de tests immunoenzymatiques utilisant tous la technique EMIT® sauf pour l'alcool (enzymologie) et pour les salicylés (photochimie).

Le bilan est ensuite complété par les screenings HPLCDAD et LC-MS. Afin de valider les chromatogrammes, $100 \mu \mathrm{l}$ d'une solution méthanolique à $10 \mu \mathrm{g} / \mathrm{ml}$ d'une benzodiazépine (RO 216295 ROCHE) sont ajoutés à $1 \mathrm{ml}$ de plasma. Le plasma est ensuite tamponné avec $1 \mathrm{ml}$ d'une solution saturée de carbonate de sodium (pH 11) avant d'être extrait par $5 \mathrm{ml}$ d'une solution de chloroforme/isopropanol 15/85. Cet extrait est séché à $40^{\circ} \mathrm{C}$ sous flux d'azote puis repris avec $100 \mu \mathrm{l}$ de phase mobile : acétonitrile / tampon formiate 15/85. $20 \mu \mathrm{l}$ sont analysés successivement en HPLC-DAD et en LCMS.

Les données HPLC-DAD sont exploitées avec la bibliothèque UV mis à disposition par WATERS. Les données LC-MS sont retraitées par le logiciel AMDIS (2) contre la bibliothèque LC-MS du laboratoire. Cette bibliothèque a été construite à partir de substances pures en solutions méthanoliques à $100 \mu \mathrm{g} / \mathrm{l}$ extraites dans les mêmes conditions que les plasmas. L'identification des composés est faite sur le couple temps de rétention/spectre de masse. Du fait de leur mauvaise réponse à la tension de cône utilisée, les benzodiazépines sont recherchées séparément par une méthode LC-MS spécifique en mode multi-SIM.

\section{Résultats}

Le protocole de screening LC-MS a été appliqué à trois types de situations cliniques : a) un bilan toxicologique systématique dans le cadre de la mort subite du nourrisson, b) la recherche de toxiques pour des patients admis en réanimation en état de coma toxique et c) à l'occasion d'une étude visant à évaluer l'intérêt du screening LC-MS lors du bilan d'admission au service des Urgences (3). Les benzodiazépines n'étant par recherchées par ce screening, leur présence n'est pas rapportée.

a) Dans le cadre de la mort subite du nourrisson, 6 cas ont été traités (tableau I). Le dépistage sanguin par analyseur immunoenzymatique a rapporté la présence de salicylés dans 3 cas, l'HPLC-DAD a mis en évidence la présence de paracétamol dans un cas et identifié des dérivés imipraminiques dans un autre cas. Le screening LC-MS a confirmé la présence d'imipraminiques en identifiant formellement l'amitriptyline et la nortriptyline.

b) Le screening LC-MS appliqué aux comas toxiques a permis d'identifier formellement de nombreux psychotropes et leurs métabolites (tableau II) en une seule

Tableau I : Résultats des screenings plasmatiques appliqués en cas de mort subite du nourrisson en fonction de la technique utilisée.

\begin{tabular}{|c|c|c|c|}
\hline Cas & Dépistage automate & MPLC DAD & HPLC-MS \\
\hline 1 & Salicylés & Salicylés & - \\
\hline 2 & Paracétamol & Paracétamol & Paracétamol \\
\hline 3 & Imipraminiques & Imipraminiques & Amitriptyline et Nortriptyline \\
\hline 4 & - & - & - \\
\hline 5 & Salicylés & Salicylés & - \\
\hline 6 & Salicylés & Salicylés & - \\
\hline
\end{tabular}


Tableau II : Toxiques identifiés dans le plasma lors des comas toxiques en LC-MS et réponse UV correspondante hors benzodiazépines.

\begin{tabular}{|c|c|c|}
\hline Cas & Molécules identifiées en LC-MS & Molécules détectées en UV \\
\hline 1 & Olanzapine, zolpidem, sertraline & - \\
\hline 2 & Alimémazine + métabolites & Phénothiazines \\
\hline 3 & Amisulpride, Alimémazine, citalopram & Amisulpride, Phénothiazines \\
\hline 4 & Lévomépromazine, cyamémazine + métabolites & Phénothiazines \\
\hline 5 & Zolpidem, Méprobamate & $-\quad+2$ \\
\hline 6 & Amisulpride, Zolpidem & Amisulpride \\
\hline 7 & Amphétamines, lidocaïne & $-\quad \therefore$ \\
\hline 8 & Méthadone + métabolites (EDDP) & - \\
\hline 9 & Benzoylecgonine (BZE) & $-\quad+1+2$ \\
\hline 10 & Flécaïne & $-\quad \cos (10$ \\
\hline 11 & Lidocaïne & - \\
\hline 12 & Amitriptyline & Imipraminiques \\
\hline 13. & Zolpidem, cyamémazine sulfoxyde & 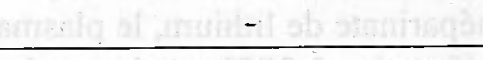 \\
\hline 14 & Zolpidem & - \\
\hline 15 & Tramadol & -4 \\
\hline 16 & Lévomépromazine + métabolites, méprobamate & Phénothiazines \\
\hline 17 & Rispéridone + métabolite & Rispéridone \\
\hline 18 & Cyamémazine + métabolites, citalopram & Phénothiazines \\
\hline 19 & Paroxétine & - \\
\hline
\end{tabular}

acquisition chromatographique. Le résultat était accessible au clinicien dans les 2 heures. Pour la plupart des molécules retrouvées, il n'existait pas d'alternative analytique plus rapide pour le dépistage de la classe pharmacochimique dans la matrice choisie.

c) Les résultats détaillés de l'étude sur l'intérêt du screening LC-MS au niveau du bilan d'admission aux Urgences a déjà été présenté (3). Sur 23 patients admis avec un indice de Glasgow inférieur à 12 , le screening avait mis en évidence dans 10 cas un psychotrope non benzodiazépiniques d'intérêt diagnostique. La proportion était plus faible lorsqu'il s'agissait de patients conscients ou non, dépistés positivement aux benzodiazépines par méthode immunoenzymatique, puisque sur 48 cas le screening n'apportait une information supplémentaire que dans 6 cas (figure 1).

\section{Discussion}

L'investigation toxicologique dans les cas de mort subite du nourrisson s'inscrit dans un cadre de semi-urgence dans la mesure où la décision d'inhumation dépend des données analytiques. D'autre part, il n'y a généralement pas d'orientation étiologique clinique. Enfin, la quantité de prélèvement est souvent faible : $200-500 \mu \mathrm{l}$. On attend dans ce cas du screening toxicologique une information qualitative rapide la plus large possible sur une vaste gamme de substances toxiques à partir d'un faible volume d'échantillon. Les dépistages immunoenzymatiques offrent l'intérêt de répondre rapidement pour de très faibles volumes et sans étapes préanalytiques importantes pour des toxiques courants (paracétamol, salicylés, barbituriques, imipraminiques). Cependant ces méthodes ne couvrent qu'un petit nombre de familles pharmacologiques. La LC-MS et l'HPLC-DAD nécessitent une étape d'extraction qui élimine nécessairement un certain nombre de molécules. Le détecteur DAD présente l'intérêt de déterminer facilement la famille chimique d'un pic détecté en revanche il est difficile d'identifier formellement une molécule d'une série métabolique à temps de rétention proches. De plus les spectres UV sont peu robustes vis à vis des composés co-éluants. Le spectre de masse permet une identification des molécules beaucoup plus formelle, notamment avec des logiciels de déconvolution. En revanche, l'identification d'un composé non répertorié dans la bibliothèque de l'utilisateur est très difficile et il n'y a pas d'informations accessibles quant à la famille chimique. 


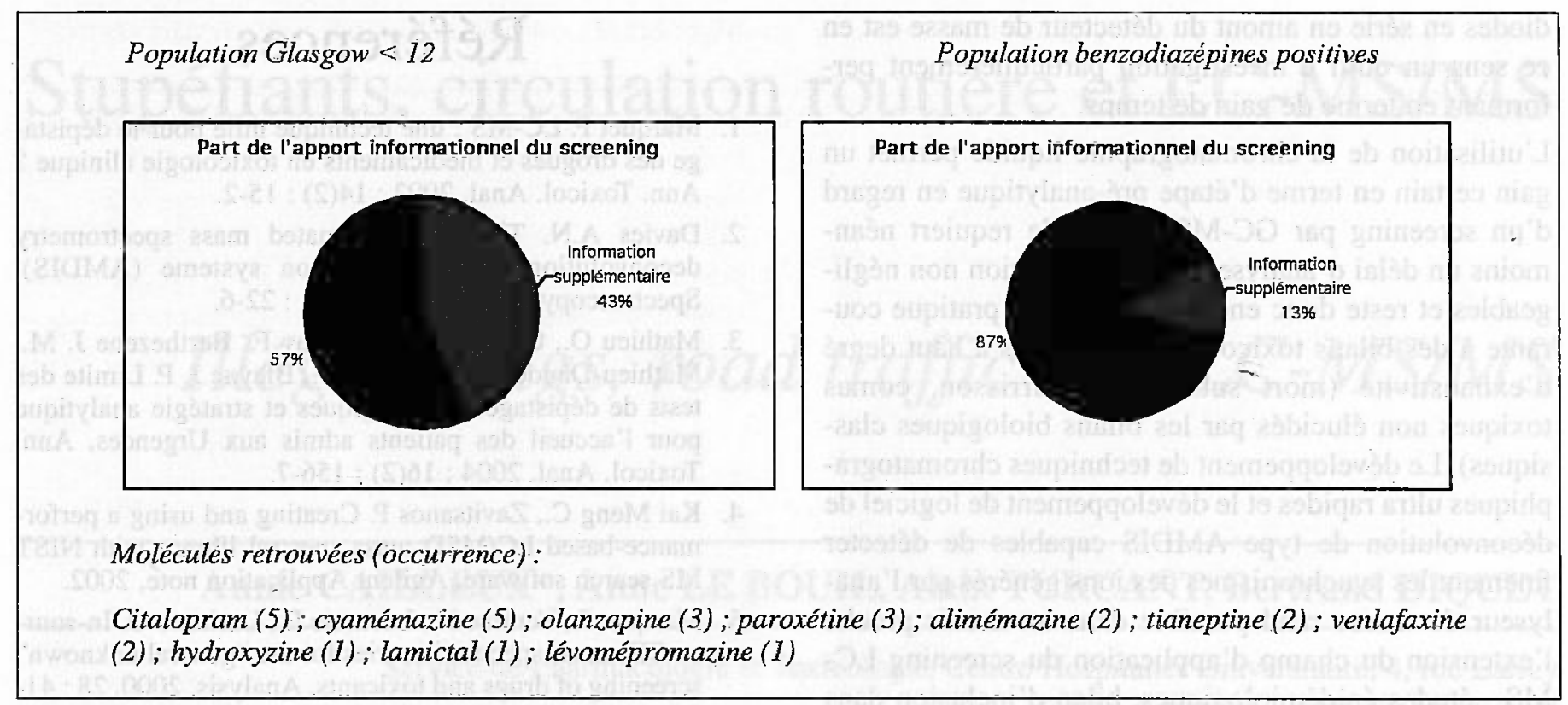

Figure 1 : Apport diagnostic du screening LC-MS par rapport au dépistage classique selon la population cible. Liste des molécules détectées.

Parmi les morts subites étudiées, l'identification directe de l'amitriptyline et de la nortriptyline (cas 3) permet d'alerter en moins de 2 heures sur la présence certaine de ces deux molécules. Les deux autres techniques employées ne permettent qu'une orientation nécessitant confirmation ultérieure et donc un délai supplémentaire pour le clinicien demandeur. En ce qui concerne les salicylés (cas 1, 5,6), le screening LC-MS en mode ES positif ne permet pas leur détection. La méthode EMIT en répondant de façon quantitative permet néanmoins d'envisager ou non une confirmation HPLC-DAD. Dans les cas présentés, cette confirmation n'offrait aucun intérêt clinique du fait d'une concentration plasmatique normale et justifiée.

Pour les comas toxiques, les circonstances cliniques sont différentes. Le travail du toxicologiste est facilité par la connaissance des signes cliniques et biologiques, parfois la connaissance des médicaments incriminés. Le volume d'échantillon n'est souvent pas un facteur limitant. L'urgentiste peut souvent se satisfaire d'une information de nature semi-quantitative. L'aspect quantitatif est souvent secondaire pour beaucoup de molécules.

L'analyse des données montre que 11 fois sur 19 , le pic UV est absent et que lorsqu'il est présent, hormis pour l'amisulpride, le paracétamol et la rispéridone, l'identification du toxique sur la donnée spectrale UV et le temps de rétention reste incertaine du fait de l'existence de métabolites chromatographiquement proches.

Parmi les molécules retrouvées en LC/MS, certaines le sont à des concentrations non toxiques que l'HPLC-
DAD aurait pu mettre en évidence si la prise avait été importante (zolpidem, olanzapine, citalopram, tramadol, paroxétine). L'information portant sur la présence de psychotropes à des concentrations thérapeutiques est intéressante pour la prise en charge du patient en cas de poly intoxication et peut permettre de confirmer les données recueillies lors de l'interrogatoire clinique. Nous avions rapporté le faible taux de fiabilité de cet interrogatoire (3). Enfin le méprobamate étant optiquement inactif n'est pas détectable en HPLC-DAD, en revanche il est identifié en spectrométrie de masse.

La possibilité d'identifier les métabolites pourrait à l'avenir, moyennant le phénotypage pharmacologique des sujets à risque, permettre d'orienter précocement une prise en charge spécifique selon un indice pronostique découlant du profil des métabolites.

Le screening toxicologique par LC-MS permet d'identifier formellement un nombre plus important de toxiques et ceci à des concentrations beaucoup plus basses que l'HPLC-DAD. La limite majeure du screening LC-MS réside dans l'obligation de constituer à une bibliothèque de spectres de masse dédiée à la plateforme. Ce travail est à la charge de l'utilisateur.

Ces deux types de screening ne sont pas en relation de concurrence mais de complémentarité puisque le croisement des résultats permet l'identification de pics inconnus : l'information sur la classe pharmacochimique obtenue par HPLC-DAD permet de rejeter certaines molécules bonnes candidates du point de vue de leur masse. Le couplage de ces deux techniques analytiques sur une même chaîne en plaçant la barrette de 
diodes en série en amont du détecteur de masse est en ce sens un outil d'investigation particulièrement performant en terme de gain de temps.

L'utilisation de la chromatographie liquide permet un gain certain en terme d'étape pré-analytique en regard d'un screening par GC-MS, mais elle requiert néanmoins un délai d'analyse et d'interprétation non négligeables et reste donc encore réservée en pratique courante à des bilans toxicologiques urgents à haut degré d'exhaustivité (mort subite du nourrisson, comas toxiques non élucidés par les bilans biologiques classiques). Le développement de techniques chromatographiques ultra rapides et le développement de logiciel de déconvolution de type AMDIS capables de détecter finement les synchronismes des ions générés par l'analyseur de masse rend possible dans un avenir proche l'extension du champ d'application du screening LCMS : études épidémiologiques, bilan d'inclusion dans une étude clinique, exploration de voies métaboliques, etc... L'obstacle majeur à la transposition du screening HPLC-DAD aux techniques ultra-rapides est l'appauvrissement de la résolution et le chevauchement des pics. Ces perturbations faussent irréversiblement la donnée analytique initiale : l'information spectrale obtenue est la sommation des absorbances des composés co-éluants et l'accès aux composants de cette somme est inaccessible. En revanche les données LCMS permettent d'accéder par retraitement à la restitution qualitative et quantitative des ions composant le signal total.

\section{Conclusion}

Après un an de recul, la LC-MS simple quad que nous utilisions essentiellement pour la quantification, nous semble un instrument performant d'identification pour des screening toxicologiques dans le cadre hospitalier.

\section{Références}

1. Marquet P. LC-MS : une technique utile pour le dépistage des drogues et médicaments en toxicologie clinique ? Ann. Toxicol. Anal. 2002; 14(2) : 15-2.

2. Davies A.N. The new automated mass spectrometry deconvolution and identification systeme (AMDIS), Spectroscopy Europe, 1998, 10/3 : 22-6.

3. Mathieu O., Gutknecht S., Barnay F., Berthezene J. M., Mathieu-Daude J. C., Benatia P., Blayac J. P. Limite des tests de dépistage toxicologiques et stratégie analytique pour l'accueil des patients admis aux Urgences, Ann. Toxicol. Anal. 2004; 16(2) : 156-7.

4. Kai Meng C., Zavitsanos P. Creating and using a performance-based LC/MSD mass spectral library with NIST MS search software, Agilent Application note, 2002.

5. Marquet P., Venisse N., Lacassie E., Lachâtre G. In-source CID mass spectral libraries for the "general unknown" screening of drugs and toxicants, Analysis, 2000, 28 : 4150.

6. Grisel F. Development of a mass spectra library with ESI and in source CID for the screening of toxicants in biological samples, Assemblée générale de la SGMS, 2002. 